the conclusions of the symposium report [*]. The HIF field is not yet sufficiently welldeveloped to define a large, multinational, central facility that would become the focus for a global-scale project akin to the International Thermonuclear Reactor (ITER) project for magnetically confined fusion. Instead, work should continue to be coordinated informally between laboratories on problems of mutual interest.

Nonetheless, a 1991 report of the US Fusion Policy Advisory Committee has established a schedule for ICF research with a driver decision for an Experimental Test Facility set for around the year 2000. In the absence of an equivalent European inertial fusion programme, this timetable is an additional reason for reinforcing nationallevel collaborations so as to keep pace with developments.

Bilateral agreements and multinational laboratories already exist for accelerator research as there is little commercial interest. For target research, it is clear that a European facility to test inertial targets is at least 10 years away. But can people in the meantime collaborate effectively while much information is classified? The report thinks classification is not a serious problem as the overall goals for key parameters are known. For reactor issues, the topics are so diverse that there must surely be plenty of room for collaboration. But would not cooperation at the national level be more effective with an European HIF programme?

\section{Worldwide Collaboration}

The symposium report offered some guidelines for international collaboration. First, it is important to avoid splintering as happened for magnetic fusion where the US alone now has ten torroidal confinement devices in nine different institutions. Second, the greatest technical challenge is to develop a low-cost driver. To illustrate the problem, an induction linac for high-gain target HIF experiments would cost $1000 \mathrm{M}$ SUS, and a RF

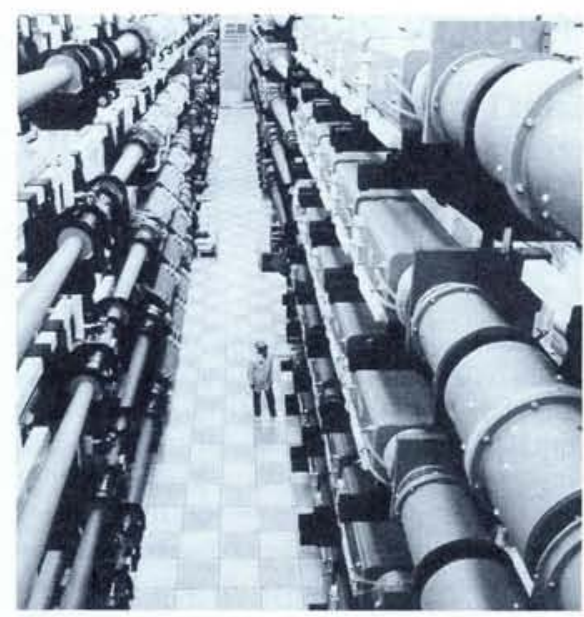

The main hall of the $70 \mathrm{~kJ}$ NOVA laser facility at the Lawrence Livermore National Laboratory, USA.

device probably even more. Moreover, system studies in Germany, the USA and Japan all show that the accelerator driver is the most expensive component of an electricity generating plant. The US has opted for a linear induction accelerator approach as it appears simpler and cheaper than approaches based on RF systems with which Europe has more experience.

The report suggests establishing common ground for collaboration by combining elements of both approaches in a new concept, namely a circulating induction accelerator proposed in 1988 by the MBE- 4 team (see page 83).

The European consensus (see below) appears to be that there should be a determined effort to collaborate on target issues. Meanwhile, one must continue development of the main accelerator alternatives for several more years before making the choice for a large demonstrator.

[*] Herrmannsfeldt W.B. et al., SLAC Report SLAC-PUB-5457 (1991).

\title{
European Programme Recommended
}

R.A. Ricci of the Laboratori Nazionale di Legnaro, Italy, and President of the Italian Physical Society summarises the results of an evaluation of heavy ion driven inertial fusion.

An Italian study of inertial confinement fusion (ICF) driven by heavy ions began in 1989 following an agreement between Ente Nazionale Energie Nucleare e Alternative (ENEA) and the Società Italiana di Fisica (SIF). In considering the possibility of a national research programme on heavy ion beam driven ICF, the evaluation was largely motivated by:

- The international scientific community's renewed interest in the physics of ICF, driven either by lasers or by charged-particle beams.

- Increased confidence in the feasibility of commercial energy generation.

- The possibility of a common effort by
Italian and German research groups in establishing a joint programme in the field, taking into account that until now European efforts have been almost exclusively devoted to magnetic confinement. This might has been justified up to now for the priority programme of the European Community, but it presently seems that new, joint initiatives concerning inertial confinement should also be considered.

\section{Starting Points}

The starting points for the study project, which also comprise the working scheme being considered for a specific Italian-German collaboration, were:
- The initial systems study HIBALL which provides the basic parameters for a reactor driver [1].

- A novel driver concept exploiting nonLiouvillian stacking as an essential technique for providing heavy ion beams of, e.g., $\mathrm{Bi}$ or $\mathrm{Ba}$, with suitable intensity and energy [2].

- Studies of driver chains for achieving the beam parameters required for ignition. These parameters have to be considered as being determined by non-Liouvillian stacking and by the reactor requirements, for both direct or indirect drive.

- Investigations of beam-target interaction, target geometries and related issues, for both direct and indirect drive, involving both computational and/or experimental studies.

\section{Status}

Reference parameters for a heavy ion ICF scheme started from HIBALL's basic data for the principle non-Liouvillian option [1]. Reviewed below is the status of various aspects of ion driven ICF following more recent evaluations reported by Italian groups.

\section{Non-Liouvillian stacking}

There is a consensus that non-Liouvillian stacking is an advanced and efficient tool for accumulating high current heavy ion beams without the usually unavoidable increase in phase space volume. A possible problem could arise in attempting to optimise the requirement of not exceeding space charge limits during high power $(\approx 500$ TW) deposition into a pellet of reduced size $(\approx 3 \mathrm{~mm})$. This is related to the choice of the accelerating scheme in which photo-ionisation is performed.

\section{Driving chain}

The driving chain can adopt several different arrangements. A generic design would be as follows [4]:

$-\mathrm{Bi}^{+}$source + RF quadrupole.

- Acceleration $(20 \mathrm{KeV} \rightarrow 1-2 \mathrm{MeV})$ including a non-Liouvillian stacking ring (NLS) connected to the FEL inducing the photoionisation of $\mathrm{Bi}^{+}$to $\mathrm{Bi}^{++}$(i.e., photo-ionisation in the storage phase) and eventually a transit-time linac if further acceleration from $1 \mathrm{MeV}$ to $1 \mathrm{GeV}$ is carried out after the NLS.

- Further storage, acceleration (transittime linac: $1 \mathrm{GeV}$ to $10 \mathrm{GeV}$ ) and pulse compression to $\Delta t=10 \mathrm{~ns}$ ).

A version which now seems to be more acceptable involves full acceleration $(1 \rightarrow 10 \mathrm{GeV})$ before the NLS with the $\mathrm{Bi}^{+} \rightarrow \mathrm{Bi}^{++}$photo-ionisation at the compression phase (see page 88 ).

The final beam parameters would be: $\approx 10 \mathrm{GeV}$ kinetic energy; pulse time $\Delta t=$ $10 \mathrm{~ns}$; $\approx 5 \mathrm{MJ}$ input energy; $500 \mathrm{TW}$ beam power; number of ions per pulse $N$ $=3.125 \times 10^{15}$ ions. Taking into account the efficiency of the NLS and losses, one 